\title{
Graduate teaching assistants' perceptions of a context-rich introductory physics problem
}

\author{
Melanie Good, ${ }^{1}$ Emily Marshman, ${ }^{1}$ Edit Yerushalmi, ${ }^{2}$ and Chandralekha Singh ${ }^{1}$ \\ ${ }^{1}$ Department of Physics and Astronomy, University of Pittsburgh, Pittsburgh, PA 15260 \\ ${ }^{2}$ Department of Science Teaching, Weizmann Institute of Science, 234 Herzl St., Rehovot, Israel 7610001
}

\begin{abstract}
Posing the same physics problem scenario in different ways can emphasize learning goals for students, such as developing expert-like problem-solving approaches. In this investigation, we examined graduate teaching assistants' (TAs') views about a context-rich introductory physics problem within a semester-long TA professional development course. The TAs were asked to list the pros and cons of a context-rich problem, rank the problem in terms of its instructional benefit and the level of challenge it might produce for their students, and describe when and how often they would use it in their own classes if they had complete control of teaching the class. We find that TAs did not find the context-rich problem to be instructionally beneficial and were unlikely to use it in their own courses. Many TAs expressed their concerns as being due to the problem seeming to be unclear or excessively challenging and time-consuming for their students. These findings suggest that there is a discrepancy between the TAs' perception of a context-rich problem and the benefits of problems posed in this manner according to the physics education research literature.
\end{abstract}

\section{INTRODUCTION AND BACKGROUND}

The desired learning goals for students in many introductory physics courses often include learning physics concepts and developing effective problem-solving skills. The cognitive apprenticeship model is a field-tested framework to support these goals. In this framework, learning takes place through a guided process in which students gradually develop self-reliance To facilitate this process, the framework includes three aspects: modeling to demonstrate the criteria for good performance, coaching and scaffolding to provide immediate feedback as students engage with problem solving, and weaning to build autonomous expertlike problem-solving ability [1]. Within this framework, the same underlying physics problem posed in different ways can facilitate different aspects of the guided process.

Prior research suggests that particular types of problems can help students develop problem-solving skills [2] and learn physics [3]. For example, a problem "type" (a problem scenario posed in a particular way) which is broken into parts may be useful in the modeling and coaching aspects as a means to demonstrate the steps one might take in formulating a logical problem solution and to scaffold student learning. Alternatively, the same underlying problem, when posed as a problem type that provides less support, can help with the weaning aspect if used after modeling and coaching.

One problem type that has been shown to be effective in helping introductory physics students become good problem solvers is a context-rich problem [4]. Context-rich physics problems are complex, lacking in structure, often provide redundant information and have real-life contexts [4]. Students who worked in groups were more likely to use effective problem-solving strategies and show positive interdependence when working on context-rich problems than when working on analogous traditional textbook problems [4]. Research suggests that students who engage with contextrich problems are more likely to think about the concepts first, and have a more positive attitude about problem solving [5]. As students become more experienced in solving context-rich problems, they show progress towards expert-like problem solving [6].
Physics graduate teaching assistants (TAs) are potential future faculty who may be responsible for making decisions about the use of different problem types in different instructional situations depending upon their perceived instructional value and constraints. Therefore, their views about the pros and cons of posing an introductory physics problem in different ways and in different instructional contexts can be useful in developing activities to improve their professional development and help them recognize the pedagogical value of posing the same problem in various ways.

A prior study was conducted about physics instructors' views about different problem types in which they were presented with the same problem variations of a physics problem (including the context-rich problem) given to the TAs in the current study [7]. It was found that the instructors generally valued different problem types intended to develop expert-like problem-solving, but they were not as likely to use certain problem types. Faculty were not very likely to use the contextrich problem type, and stated that their reluctance was either to avoid stressful situations for students since these problems are complex and ill-structured or because they did not want to change their traditional teaching practice [7].

Prior research has investigated TAs teaching beliefs and practices. For example, TAs often struggle with grading in a way that encourages students to show their work, which can in turn improve their learning from problem-solving and help them learn from their mistakes [8-[10]. Another study involving TAs' beliefs shows that the TAs were unlikely to identify features in example problem solutions that the research literature describes as supporting learning goals for students [11].

In the study presented here, TAs in a TA professional development course were asked to reflect upon the same five problem types for an introductory mechanics problem scenario posed to the instructors in the earlier study [7]. Here we focus on TAs' initial views about a context-rich introductory physics problem and investigate the following research questions: (1) How challenging and instructionally beneficial do TAs perceive a context-rich problem? (2) If TAs had complete control of an introductory course, would they be likely to use a context-rich problem? (3) Why do TAs perceive a context-rich problem the way they do? 
TABLE I: The most commonly listed pros/cons of a context-rich problem and the percentages of TAs who listed them. Some TAs listed more than one of the following or other pros/cons not listed here. However, other pros were negligible and the overlap of the two cons listed below represents only $5 \%$ of all TAs.

\begin{tabular}{|l|l|l|l|}
\hline Code & Definition & Examples & Percentage of TAs \\
\hline (Pro) real & $\begin{array}{l}\text { real life scenario; can help students } \\
\text { relate physics to their lives }\end{array}$ & $\begin{array}{l}\text { gives insight to how physics laws and principles are } \\
\text { ingrained in our lives" }\end{array}$ & $47 \%$ \\
\hline (Con) time & time-consuming & "takes up a lot of time" "can take too long" & $33 \%$ \\
\hline (Con) unclear & problem is vague and/or confusing & $\begin{array}{l}\text { "can be ambiguous; not sure what to do" "hard to } \\
\text { decipher" }\end{array}$ & $42 \%$ \\
\hline
\end{tabular}

\section{METHODOLOGY AND DATA COLLECTION}

Participants: A total of 97 TAs participated in this study during 4 different years. Participants were physics graduate students who were enrolled in a mandatory TA professional development course that met once per week for 2 hours for an entire semester. The TAs were responsible for approximately one hour of homework each week pertaining to the professional development course. They concurrently had teaching responsibilities (recitation or lab instruction and most were assigned to also help students in the physics tutoring center).

Data Collection Tools and Artifacts: The data collection tools consisted of five introductory physics problem types that had been developed previously [7]. The problem types were designed for the same introductory physics problem scenario in mechanics. These problem types included two different versions of broken into parts problems, a multiple-choice problem, a context-rich problem and a traditional textbook version of the problem. Here we focus on the context-rich problem (see Figure 1). This problem requires that students first construct a concrete question and then solve the problem using the relevant information provided (extraneous information is also included and the problem requires explicit calculation after formulating the problem). Based upon our research questions, the TAs were asked to answer questions about these problem types on a worksheet, a partial sample of which can be found in Figure 2. Among the entries shown in Figure 2 TAs were to include at least one pro and one con for each problem type, and include a way in which each problem type could be used. Data were collected over four different years. In the most recent year's worksheet, which was given to 27 of the 97 TAs in the study, TAs were also asked (in addition to the entries included in Figure 2) to rank the problem types on their instructional benefit (i.e., how instructionally beneficial the TAs judged each problem type to be), the level of challenge (i.e., how difficult the TAs judged each problem type to be for students), how much they liked the problem types, and the likelihood of them using the problem if they had complete control of the choice of the problem types to use. For example, a TA who ranked a problem 1 for "challenging" judged this problem to be the least challenging for students; a 5 for "challenging" would mean that the TA perceived it to be the most challenging out of the five problem types. The rankings allowed us to investigate research questions 1 and 2. Furthermore, throughout all four years, TAs were asked to list pros and cons of the problem types. These pros and cons were useful for investigating why TAs ranked the problem types the way they did (research question 3).

\section{Problem C}

You are working at a construction site and need to get a $3 \mathrm{lb}$. bag of nails to your co-worker standing on the top of the building ( $60 \mathrm{ft}$. from the ground). You don't want to climb all the way up and then back down again, so you try to throw the bag of nails up. Unfortunately, you're not strong enough to throw the bag of nails all the way up so you try another method. You tie the bag of nails to the end of a $2 \mathrm{ft}$. string and whirl the string around in a vertical circle. You try this, and after a little while of moving your hand back and forth to get the bag going in a circle you notice that you no longer have to move your hand to keep the bag moving in a circle. You think that if you release the bag of nails when the string is horizontal to the ground that the bag will go up to your co-worker. As you whirl the bag of nails around, however, you begin to worry that the string might break, so you stop and attempt to decide before continuing According to the string manufacturer, the string is designed to hold up to $100 \mathrm{lbs}$. You know from experience that the string is most likely to break when the bag of nails is at its lowest point.

FIG. 1: The context-rich problem posed to the TAs

\begin{tabular}{|c|c|c|c|c|c|c|}
\hline \multirow{2}{*}{$\begin{array}{l}\text { Problem } \\
\text { Type }\end{array}$} & \multirow{2}{*}{$\begin{array}{l}\text { What are the FEATURES } \\
\text { of the problem? }\end{array}$} & \multirow{2}{*}{$\begin{array}{l}\text { What are the } \\
\text { PROSICONS of the } \\
\text { problem? }\end{array}$} & \multicolumn{4}{|c|}{ Would you use this problem as. } \\
\hline & & & $\begin{array}{l}\text { a HW problem? } \\
\text { Why or why } \\
\text { pot? }\end{array}$ & $\begin{array}{l}\text { DQ QUZZ problem? } \\
\text { Why or why not? }\end{array}$ & $\begin{array}{l}\text { an EXAM problem? } \\
\text { Why or why not? }\end{array}$ & $\begin{array}{l}\text { a small GROUTP problem } \\
\text { solving session? } \\
\text { Why/why not? }\end{array}$ \\
\hline Problem C & $\begin{array}{l}\text { Practical apdica- } \\
\text { tion problem: } \\
\text { No diagram. }\end{array}$ & $\begin{array}{l}\text { Pros: Connect } \\
\text { mowuledige to } \\
\text { practical application } \\
\text { Cons: To lang } \\
\text { description. }\end{array}$ & $\begin{array}{l}\text { No, tod } \\
\text { many une } \\
\text { - cessany } \\
\text { descriptions }\end{array}$ & $\begin{array}{l}\text { No, takes } \\
\text { too ruch time } \\
\text { to read. }\end{array}$ & $\begin{array}{l}\text { No, tales } \\
\text { too much time }\end{array}$ & $\begin{array}{l}\text { No, takes ton } \\
\text { much time }\end{array}$ \\
\hline
\end{tabular}

FIG. 2: Part of a sample worksheet given to the TAs

Data Collection in the TA Professional Development Course and Later in Individual Interviews: TAs were given the problem types and worksheets in the professional development course in the middle of the semester when they had some teaching experience in order to elicit their initial ideas about different problem types. They were asked to answer worksheet questions under the assumption that they had complete control over the introductory physics class, including control over problem types chosen for various purposes. The worksheet was completed as part of a homework assignment. Nine participants who had taken the TA professional development course earlier volunteered to be interviewed in a one-on-one setting using a think-aloud protocol. Due to availability of researchers and interviewees, interviews took place approximiately one semester after the activity in the TA professional development course. These interviews more deeply probed the TAs' reasoning for their responses.

Coding TA Responses: Two of the researchers met weekly to help identify appropriate coding categories for pros/cons for all four years of data; agreements on these were reached through discussion. The inter-rater reliability was examined for the coding of the pros/cons for a subset of the data (i.e. one of the four years) that was coded, and the average Cohen's kappa for that subset was calculated to be $\kappa=0.982$. 
Three most common pros and cons for the context-rich problem, along with their definitions and examples from TA worksheets are included in Table [ We note that for the context-rich problem, some TAs' responses could not always be appropriately coded as a particular pro or con because they were sometimes negative but non-specific in nature. For example one TA response was, "never assign this."

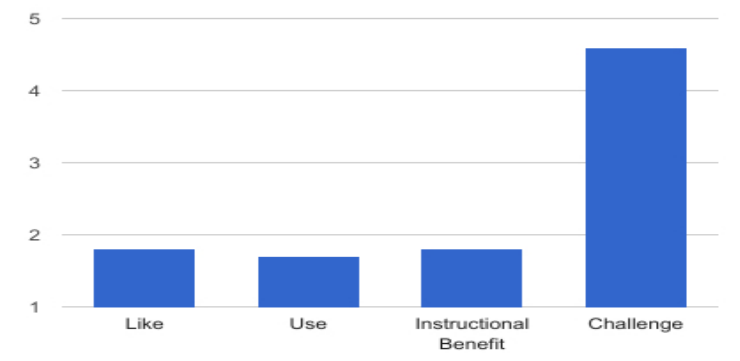

FIG. 3: Average rankings of the context-rich problem most recent years worksheets.

\section{RESULTS}

Context-rich problem ranked lowest for like, use, and benefit, but highest for level of challenge for students: Figure 3 summarizes the rankings for the context-rich problem in all four categories. On average, the context-rich problem received the lowest ranking of all problem types in the three categories "like," "use," and "instructional benefit". On the other hand, the context-rich problem was ranked, on average, the highest for "challenge" out of all five problem types the TAs were given. Given the extreme rankings of the contextrich problem, we examined the pros and cons the TAs listed as well as feedback gained during individual interviews to look for reasons for why they perceived the context-rich problem the way they did, in response to research question (3).

TAs viewed the context-rich problem as unclear and time consuming: The pros and cons in written responses as well as the interview data elucidate possible reasons for why the TAs ranked the context-rich problem low for "like," "use," and "instructional benefit," and high for "challenge." Table I shows the most common pros and cons mentioned by TAs in written responses. The most common pro was "real." (i.e., relatable to real-life scenario). One TA put it succintly: "Connects to daily life." TAs seldom mentioned any other pro, and many TAs did not list any pros at all even though they were asked to list at least one pro. The inability to come up with a pro for the context-rich problem supports the fact that TAs did not perceive this type of problem to be instructionally beneficial. The lack of perceived pros may also explain the low average rankings of TAs on "like" and "use" for the context-rich problem. Moreover, interviews suggest that the pro "real" may not be perceived as compelling enough to outweigh the negative light in which the TAs viewed the contextrich problem overall. For example, one interviewed TA stated, "There's some redeeming elements to it, like I like that this frames it from the perspective of the student, so they can think about what they see and feel... So it's not all bad, but...I would tend not to use it at any level. I just don't think its an effective problem." This TA appears to recognize some "redeeming elements" in the real-life aspects of the problem, but qualifies this pro by stating that this is not enough of a reason for him to use a problem like this in his own classes. Additionally, the remark about it not being effective appears to speak to this TA's low opinion of the problem in terms of its instructional benefit. Other TAs also had similar views.

Table I shows that the most commonly stated cons were "unclear" and "time." "Unclear" refers to TA responses which described the problem feature as confusing (e.g.,"Overly confusing and frustrating”) or lacking in clarity or explicit question (e.g.,"The point of the problem is not clear"). In fact, the con "unclear" appears to be one major reason for why the TAs thought that the context-rich problem was highly challenging. In particular, the majority of TAs who ranked the context-rich problem as the most challenging also mentioned "unclear" as a con. The TAs who listed the con "unclear" had a variety of reasons for why they perceived this problem to be lacking in clarity, and often the same TA stated more than one reason for why he/she felt the problem was unclear. Written responses and interviews suggest that one reason the context-rich problem was often seen as unclear was that the TAs felt it was verbose with too much extraneous information that their students will have difficulty making sense of. For example, one interviewed TA first described the problem as having "too much detail," and then went on as follows, "it's very good that it has a story to it but the story is too much and the science is not enough. Because this is a physics class and... it looks more like a story than a physics problem." It is interesting that the TA appears to view the narrative aspect of the problem (i.e., the "story") as separate from the "science" of the problem. Further discussion with the TA suggests that he preferred textbook problems and viewed the detailed descriptive narrative in a physics problem as being outside of the scope of what should be part of a "usual" physics curriculum. Another TA also remarked, "I think... intuition in being given a word problem and knowing how to translate that into math is the hardest part." Further discussions with this TA suggest that he agreed that formulating the problem (i.e., translating the problem from words to quantitative expressions) may potentially be useful for students. However, he then stated that he would not use this type of problem in his own class due to the fact that it was unclear and challenging for students and his students would not know how to solve this type of problem.

Another reason many TAs found this problem to be "unclear" was that there was a lack of explicit question in the problem. For example, one interviewed TA explained that the lack of an explicit question made the problem confusing: "This one is very vague. It's not asking any question, so the student might get confused about what they're supposed to do...." This type of sentiment about the lack of explicit question and the problem being "vague" or a source of confusion for students was commonly mentioned by the TAs both in written responses and interviews. For example, another interviewed TA who described the problem as "unclear" explained, "at least if you give a student a problem where they know what they need to do, they can ask you questions about that, but I can imagine students sitting there saying 'I don't know where 
to start." This TA suggested that he felt that his students will be stuck from proceeding because, without being able to discern a clear goal, they will neither be able to make sense of the problem nor be able to reach out for support from the TA or instructor as they will not know what questions to ask them. Similarly to this TA, many TAs connected a perceived lack of clarity to the fact that the context-rich problem does not ask an explicit question. Another TA stated, "It is challenging... because the wording of the problem is vague. I don't even really see an explicit question that it's asking." This TA (and many others) explicitly made a connection between the level of challenge and the lack of a concrete question posed. This TA also indicated he would not use this type of problem under any circumstance and appeared not to recognize the instructioal benefit of requiring students to determine the question that is being asked of them. However, since all real world unsolved problems require formulation of the question before solving them, learning to formulate the question is an important problem-solving skill.

In addition to the con "unclear," another common con was "time." "Time" was the category used for TA responses that indicated that solving this problem would be too time consuming for students ("It may take a while for the student to interpret the problem into a mechanics problem") and/or did not make the best use of time ("Takes time reading things that are not directly helpful for solving the problem."). The con "time" may also have contributed to TAs' hesitation in using this problem in their own classes. Of the TAs who ranked the context-rich problem the lowest in terms of "use", the majority listed "time" as a con for this type of problem. A TA's reluctance to use a context-rich problem due in part to time constraints is evident in the following comment during the interview: "The student will take much more time to solve this... I think the student has to read this question so many times more to understand it. ...I won't use this at all." We note that this TA identifies both the time it will take the student to solve the problem and also the time required to read and understand the problem statement as being problematic with regard to the use of this problem. It is interesting that this TA and others did not envision any situation in which a context-rich problem could be used effectively, e.g., in a collaborative group problem solving session. That the ranking for "use" was the lowest of all problem types indicates a reluctance to use a contextrich problem. Furthermore, even though the worksheet instructions included a directive to think of at least one way in which each problem type could be used, over $20 \%$ of the TAs stated that they would never use a context-rich problem in any way and listed no pro even when asked for at least one pro.

Thus, written responses and interviews suggest that overall, the TAs appear to have a negative opinion of the context-rich problem. Moreover, it is interesting to note that the contextrich problem elicited a strong negative response from many TAs. Some TAs stated:, "Absolutely does not help the students at all," "meaningless," and "It sucks." In summary, for the context-rich problem, there were more cons listed than pros and the cons were often strongly worded, which appears consistent for the very low rankings it received in categories related to use, like, and instructional benefit.

\section{SUMMARY AND CONCLUSIONS}

Our findings suggest that TAs did not, in general, value a context-rich problem. Many TAs felt that the context-rich problem was too challenging to be of any instructional benefit. The majority of TAs were not likely to use it in their classes. The reasons for TAs' views often pointed towards the context-rich problem being unclear and difficult to make sense of for their students as well as being time-consuming. A number of TAs mentioned the lack of a clear-cut question, wordiness, and extraneous information of the context-rich problem as being problematic. The TAs also identified the time required to parse the information and formulate the problem as being major drawbacks to using a context-rich problem. However, many of the perceived drawbacks of context-rich problems mentioned by TAs are positive aspects of contextrich problems per the research literature [4, 5]. For example, the lengthy narrative without a clear question can develop students' ability to differentiate what information is important, identify the concepts, and formulate the problem. Yet these beneficial aspects were perceived by TAs in a negative light.

Leaders of TA professional development programs can use the findings of this study to help TAs reflect on the benefits of context-rich problems. For example, professional development programs can help TAs reflect on the use of contextrich problems in collaborative group problem solving settings to help students become good problem solvers. The use of a context-rich problem in collaborative problem solving fits within the framework of the cognitive apprenticeship model in the coaching and scaffolding aspect, with the support provided by the peers. TAs may also be asked to reflect upon how and when the peer support could be weaned so that students could solve such problems on their own.

\section{ACKNOWLEDGMENTS}

We thank the NSF for award DUE-1524575.
[1] A. Collins, et. al.,Center for the study of reading, Technical Report No. 403 (1987)

[2] C. Singh, AIP Conf. Proc. (2004). doi:10.1063/1.1807283

[3] S-Y Lin, C. Singh, Phys. Rev. STPER 11, 020105 (2015)

[4] P. Heller \& M. Hollabaugh, Am. J. Phys. 60, 7 (1992)

[5] C. A. Ogilvie, arXiv:0809.1081
[6] P. Antonenko, et. al., arXiv:physics/0701284

[7] E. Yerushalmi, et. al. Phys. Rev. STPER 6, 020108 (2010)

[8] E. Yerushalmi, et. al. (2015) doi: 10.1119/perc.2014.pr.068.

[9] C. Henderson, et. al., (2015) doi: 10.1119/perc.2014.pr.024.

[10] E. Yerushalmi, et. al. (2016) doi: 10.1119/perc.2016.pr.097.

[11] S-Y Lin, et. al. Phys. Rev. 9, 010120 (2013) 